1889 , the sinus remaining from the wound, which was coated with phosphatic deposit, was thoroughly opened up, the lower part pared, the upper part scraped with a sharp spoon until the urethra was reached, and found quite clean. After this the treatment consinted in washing out the bladder twice a day with a tolerably strong solution of nitric acid, and gradually the sinus closed; but still a piece of "grit" occasionally stuck in the prostatic urethra and caused trouble, until it was washed away by the stream of urine. The man left the hospital on April 11th and returned to his work. The sinus was soundly healed, but he came to the hospital two or three evenings a week to have the bladder washed out, as small pieces of "grit" were still passed occasionally. "Throughout the summer he attended once or twice a week for this purpose.

Some time in August the house-surgeons on two or three occasions felt some obstruction (apparently calculous), which occasioned some difficulty in passing the catheter; then that trouble ceased. But towards the end of September he had such a severe attack of cystitis that he was obliged to give up work, and on Oct. 11th, 1889, he was again admitted to hospital, suffering from all the symptoms which had been noticed a year before, only in a minor degree. With the sound, a fair-sized calculus was found in the same position as on the former occasion. In consequence of the patient having a severe attack of urticaria, operation was postponed to Oct. 24th (a year all but a week from the first lithotomy). Under chloroform, the stone was felt to be quite loose in the bladder. The incision was made along the line of the former operation; the bladder was readily entered, and with the finger a soft calculus (about the size of a walnut) was felt. As soon as it was grasped with the forceps it broke up, and only about half of the mass (with its nucleus) was removed; the rest was removed by forceps, scoop, and prolonged irrigation of the bladder with warm boracic acid solution. The only trouble afterwards was the chronic cystitis with continued tendency to phosphatic deposits about the wound. It appeared likely that this condition would again prove a serious drawback; when it was decided to try the internal administration of saccharin in addition to the other methods of treatment, and in two or three days the character of the urine completely changed it became sweet, clear, light amber.coloured, free from deposits, and so it has continued ever since. The sinus closed in about a month, and the man left the hospital on Dec. 18th.

Remarks by Mr. C. Puzey.-There are two distinct matters in the history of this case which, I think, call for special comment. One is the reason why the median bilateral operation was selected for the first operation; the other is the cause of, or the method of, formation of the second calculus. With regard to the first point, I may say that it was not until the man was fully ancsthetised that I knew that I had to deal with a large intra-vesical calculus. On previous examinations (without chloroform) by myself and colleagues, it had appeared that the stone was fixed in the situation of the prostate gland (certainly the sound did not fully enter the bladder), and it appeared that we had to deal with a large prostatic calculus. This is no doubt to be explained thus: the hypertrophied and contracted bladder so spasmodically grasped the stone, that it was impossible for the sound to do more than insinuate itself a little way between the stone and the anterior wall of the bladder. Under chloroform, all muscular spasm was overcome, and the result of the examination was quite different. The same thing was noticed on the occasion of the second operation, and it affords a hint as to the desirability of "sounding" under anæsthetics in all cases involving doubtful diagnosis. As it was I had to make a rapid decision. The extreme stoutness of the man, and his large hernia, would have made the supra-pubic operation most difficult, especially as his longcontracted bladder would probably be incapable of forcible distension. The contracted state of the bladder, and the extremely severe cystitis which existed, together with the size of the calculus contraindicated litholapaxy. With regard to lateral lithotomy, I admit that I was influenced against that by the fatal difficulties which I had seen encountered by a first-rate operator in a remarkably similar case. The depth of the wound rendered it impossible for the surgern to reach the bladder with his finger; the operation was extremely prolonged, accompanied with troublesome hæemorrhage, and the patient never rallied. There is no doubt that the median incision affords the shortest route to the bladder. Then with regard to the formation of the second calculus : its nucleus was of a peculiar shape, resembling the shaft of a small metacarpal bone; its length was one inch and three-eighths, its greatest width three-eighths of an inch; its composition phosphatic. It appeared to have been monlded in the prostatic urethra, and had been broken into two pieces. The history of the formation of the second stone was doubtless this: The slowly increasing calculus which had formed in the prostatic urethra was dislodged, broken across, and pushed into the bladder by the catheter, and then it rapidly increased in growth by the heaping up of ammoniacal phosphates and the other products of cystitis. As to the source of the enormous quantity of small con. cretions which were passed for weeks, or rather months, after the first operation, I can only imagine that they had lined the rugæ of the long-contracted bladder, and that, as we day by day washed out and distended that viscus, these deposits were dislodged. They were certainly independent formations, and not fragments of a fractured calculus.

\section{DERBYSHIRE GENERAL INFIRMARY.}

\section{GOITRE IN A BOY CAUSING SOFTENING OF TRACHEAL} CARTILAGES ; TRACHEOTOMY ; DEATH.

\section{(Under the care of Mr. CuRGENTEN.)}

THE operation of tracheotomy in cases of acute goitre is sometimes attended by difficulties which are almost insuperable, and to overcome them the greatest skill and care on the part of the operator are demanded. The urgent attack may come on at night unexpectedly There may be no time to give chloroform, the surgeon may be single-handed, the dyspncea urgent and distressing, the patient possibly half dead from asphyxia, but retaining sufficient conscionsness to move and resist the incision in his neck. The hæmorrhage from the distended veins is free, and increases as the incision is deepened; the trachea may be difficult to find because displaced, altered in shape and consistency, and so far from the surface that the ordinary tubes will not reach it, or remain in situ, should they be of sufficient length. The dyspnœa in these cases appears to be caused by compression of the trachea, and the note of its condition as observed post mortem in this case, "softened and partially absorbed," is important. For the following notes we are indebted to Mr. Chas. H. Tayler, house surgeon.

A. $\mathrm{H}-$, a lad of fourteen, was admitted into this infirmary on Dec. 2nd, 1889, suffering from extreme dyspnœa. Both lobes of the thyroid were seen to be slightly enlarged, and the isthmus very much increased in size. Inspiration was more interfered with than expiration. He stated that the swelling began about five months previously, and had only interfered with his breathing the last few days. The boy was put to bed and iced cloths applied to the throat and his breathing became easier. Early on the following morning dyspncea came on again, and tracheotomy was decided upon. Assisted by Dr. Kirkpatrick, the assistant house surgeon, Mr. Tayler carefully cut down in the middle line in the small space at his disposal between the isthmus and the upper edge of the sternum to a depth of about one inch, but could neither see nor feel anything corresponding to the trachea. The bremorrhage was excessive, and the patient's condition was becoming desperate, so Mr. Tayler deepened his incision, still keeping carefully in the middle line. So low down the throat was it necessary to carry the dis. section owing to the encroachment of the isthmus, that in the lower angle of the wound, the aorta could befelt pulsating. Again a finger was inserted, but neither the trachea nor ever movement of any structure could be felt during his attempts at inspiration, which were becoming gradually fewer and feebler. Examination of the left side disclosed nothing, but at last low down to the right side what seemed to be a knuckle of cartilage was discovered. Passing the scalpe along the finger, this was notched and in and out rushed air. Dilating forceps were then introduced and an attempt made to introduce a bivalve trachea tube; but no ordinary tube would reach through the opening in the trachea and remain in, so displaced was the trachea. Finally a full sized silver catheter was passed in, and by suction and 
inflation the boy was brought round completely. Four hours afterwards he suddenly pulled out the catheter and nearly died before it could be replaced. Several attempts were made to substitute some better tube for the catheter, but those used were all too short to remain in. The great difficulty with the catheter was in preventing the eye becoming choked with mucus. Expecturation increased in quantity, and pneumonia set in, from which he died, two days after the tracheotomy had been performed.

Necropsy (permission could only be obtained to examine the throat). - The trachea was found pushed over to the right side and rotated on itself. The cartilaginous rings, from the fifth to the eight inclusive, were softened and partially absorbed, thus allowing the trachea to collapse.

Remarks by Mr. TAYLER.-There are several points of interest in this case. In the first place, the boy was not a native of Derbyshire, he having only lived a few years in this district. Then it is comparatively rare for goitre to affect a boy, and before the age of puberty. Lastly, it is uncommon for so slight an enlargement of the thyroid gland to cause such serious symptoms as he presented.

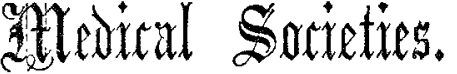

\section{ROYAL MEDICAL \& CHIRURGICAL SOCIETY.}

\section{Analysis of 964 Cases of Operation for Calculus in the Bladder.}

AN ordinary meeting of this Society was held on March 11 th, the President, Mr. Timothy Holmes, in the chair. Sir HeNRY THOMPSON communicated a paper containing an analysis of 964 cases of operation for calculus in the bladder by lithotomy and lithotrity, with remarks. The calculi removed were exhibited to the Society. The paper commenced by referring to the author's first record of 500 cases of operation for stone in the bladder in the adult male presented to the Royal Medical and Chirurgical Society in 1878 (vide Transactions, vol. lxi., p. 159). He now broughi forward an additional series of $\mathbf{4 6 4}$ cases, making a total of 964 , including cases of women and children. Of these, 101 cases occurred in hospital practice (University College) and 863 were treated in private. Besides these were a few cases of foreign bodies \&c. removed by operation. The total number of children was 17 , of women 14 , leaving the number of operations on the adult male by all methods 933 . Of every one of these the author possessed full notes made at the time, and exhibited every calculus (with very few exceptions) in a cabinet with numbered cells. A catalogue corresponding thereto contained a brief epitome of facts relating to the case, and the name of the medical man who sent it, with the subsequent history when obtainable. A brief account of the progress of lithotrity, from the an thor's earliest case (1854) until the present time, and the influence of it on his practice, was given, commencing with the method of Civiale at that time, the introduction of the aspirator for débris by Clover in 1865, and the gradually increased use of it during the next twelve years. Then followed the introduction (1878) of the great and important principle by Professor Bigelow of employing one sitting instead of several, and removing the whole of the débris at the same time, which the author at once adopted. The results of this practice were shown, on comparing his own 325 cases of the operation by a single sitting with the 475 by multiple sittings, prior to the date named, to have reduced the mortality nearly one half. The first remark. able fact obtained by analysis of the entire series was the following-namely, that stone in the bladder was not more common in children than in adult patients, as formerly supposed, due to the experience of the malady met with in hospital practice. Probably it might be so among the poorer class of the population; but it was far otherwise among those whose circumstances permitted a dietary unlimited in kind and quantity, as regards both food and drink. The following table afforded warrant for this assertion

\begin{tabular}{|c|c|c|c|c|c|}
\hline $\begin{array}{l}\text { 3elow } 16 \\
\text { years. } \\
3 \ldots\end{array}$ & $\begin{array}{l}16 \text { to } 24 \\
\text { years. } \\
\cdots \quad 8\end{array}$ & $\begin{array}{r}25 \text { to } 50 \\
\text { years. } \\
. \quad 89\end{array}$ & $\begin{array}{l}51 \text { to } 70 \\
\text { years. } \\
.565 .\end{array}$ & $\begin{array}{c}\text { Above } 70 \\
\text { years. } \\
. .184 \ldots\end{array}$ & \\
\hline
\end{tabular}

The mean age of the entire adult male cases was about sixty-two years and a half. The greatest age at which an operation had been performed was ninety-one years (lithotrity). The largest calculus was of uric acid weighing fourteen ounces. The next important observation affirmed the value of the principle, Iong ago in sisted upon by the author, of finding the calculus at the earliest date possible for the elderly adult male, in whom it so frequently occurred. Two cogent reasons existed for this: first, the stone when small was easily re. rnoved, and very little risk attended the operation; secondly, at this stage of calculus production the re-formation of an acid calculus (uric or oxalic) could almost invariably be prevented by strict dietetic precautions. A strong array of evidence was adduced in support of the foregoing. Another topic treated at length related to the choice of operation, whether by knife or by lithotrite. In the entire adult series of 933 cases 800 were by lithotrity, 115 by perineal lithotorny, and $18 \mathrm{by}$ supra-pubic lithotomy. The last important subject related to the nature and causes of fatal issue following operation, and these were given in a tabular form under ten separate heads. A brief section closed the report, pointing out the most unusual cases met with, such as exfoliations of bone from the hip into the bladder as a nucleus of stone \&c. Examples of encysted calculus of remarkable size and structure, besides curious foreign bodies introduced by accident or design, were related.

Mr. BRYANT said he felt uncomfortable in approaching the subject when the question of treatment was not open to discussion. It was interesting to observe how the author had been gradually swayed in his practice by the introduction of newer methods. He remarked on the enormous advance made by the introduction of the operation of lithotrity at one sitting by Bigelow, which he considered the most important since the question of operation for stone had been dealt with at all by surgeons. Referring to the change in fashion with lithotomy, he said it was evident that the lateral was gradually being put aside for the suprapubic method. He asked under what circumstances Sir Henry Thompson would prefer the supra-pubic method to lithotrity in adults, and also under what circumstances he would adopt the supra-pubic operation in preference to the perineal one in children. All would agree with the importance of dealing with a stone at the earliest possible stage, and both physicians and surgeons were now in the habit of examining the bladder at once if there were signs of persistent irritation.

Mr. Buckston Browne wished to remark on a point which had nuch exercised his mind. He scarcely knew how to classify the various kinds of calculi removed. The author had laid it down that removal of phosphatic con. cretions was not to be considered as an operation of lithotrity for the purposes of statistics, and he had fixed an arbitrary limit of twenty grains, to concretions below which weight the name of stone was not to be applied. Other surgeons, on the other hand, had classed as calculi stones which weighed only three or four grains. He showed a box containing nearly 300 calculi, their total weight being 170 grains, the whole of which he hal removed from a bladder through a tube, a lithotrite not being used. How was such a case to be classified if one only dealt with them nuder the heads of cutting and crushing. He thought the time had come when three designations should be used for cutting, crushing, and washing out; lithotomy and lithotrity for the two former, while the term "litholapaxy" should be reserved to imply the removal of a stone or stones without the use of the knife or lithotrite.

Mr. HOLMES said that one of the most important points raised was the possibility of detecting and removing a stono early, and another equally important and gratifying point was the possibility of guaranteeing the patient from the risk of recurrence of stone if he would adhere to a properly regulated diet. The reintroduction of supra-pubic lithotomy made it possible to remove a mas. 3 of enlarged prostate if that condition occurred as a complication of stone. It was satisfactory to notice that in a large proportion of cases after lithotrity the cure seemed to be definite. He asked whether the children of the poor met with in hospitals were better dealt with by lithotony or lithotrity; he had in these cases such ample reason to be satisfied with the former that he had never tried the latter; he believed, however, that the results of lithotrity in children had been exceedingly favourable in India.

Sir HENRY ThOMPson, in reply, said that he was very glad to have had the opportunity of personally bringing the subject before the Society. The question whether the 